\title{
A Contingent Technology Innovation Diffusion Model and Simulation
}

\author{
Guo-Zhong Yang ${ }^{1, a,{ }^{*},}$ Zhi Yan ${ }^{2, b}$ \\ ${ }^{1,2}$ School of Business, Central South University, Changsha 410083, China \\ ay5735@126.com, b153048268@qq.com \\ "Corresponding author
}

\begin{abstract}
Keywords: Technology innovation diffusion, Contingent diffusion model, Bass model, Base product, Attached product.
\end{abstract}

\begin{abstract}
Considering the dependence between innovation product and its attached products, the paper tries to relax the assumptions of Bass model and establishes a contingent technology innovation diffusion model based on dynamic time. And then its simulation is operated by software. Research result shows that attached product, which is late to diffuse than its base product, will spread quickly at the beginning of diffusion and it is easy to accept by consumers, finally all adopters of base product are potential adopters of attached product. The author also proposes some suggestions for accelerating the speed of technology innovation diffusion.
\end{abstract}

\section{Introduction}

Schumpeter, who's the founder of technology innovation, regards technology innovation of large-area or large-scale imitation as technology innovation diffusion [1]. Rogers thinks that the technology innovation diffusion is a process that the innovation spreads in the members of a social system through specific channels during a period of time [2]. The current papers address both streams of research, which research theoretical framework by making a special study of theory; find the rules of technology innovation diffusion by modeling and empirical analysis.

Bass model [3] combines with Fourt-Woodlock model [4] and Mansfield model [5]. Many scholars validate the model by using various data, and successfully predict the development trend of new technologies and new products. With the development of science and technology, the diffusion between product and technology is more and more closely linked. Scholars, who study the product diffusion model, relax appropriately the assumptions given by Bass to make it more consistent with the diffusion characteristics between products. Such as Norton and Bass [6] propose a diffusion theory model of adoption and substitution for successive generations of high-technology product; Perterson and Mahajan[7] study innovation diffusion in a dynamic potential adopter population; Bucklin and Sengupta[8] point out that the effect produced by conjugate diffusion is stronger than innovation itself. Yang Guozhong and Liu Congmin [9] formulate a model of multi-technology innovation diffusion; Thakurta and Roy [10] think that the technology penetration velocity affects the diffusion of products in tissues.

The characteristic of attached product diffusion is that the diffusion of a technology or product base on the diffusion of another technology or product, where we define the former as attached product and the latter as fundamental product, its development scale is also affected by the fundamental technology or product diffusion [11]. In the research of attached product diffusion model, traditional models, such as Bass model, have not considered the dependence between the attached product and fundamental product; Peterson and Mahajan model (PMM) considers the contingent diffusion characteristics, but the model is only suitable for attached products diffusion beginning with fundamental product diffusion at the same time. However, diffusion of attached product is generally late than that of the fundamental product. Additionally, PMM assumes the number of potential adopters of attached product is equal to cumulative adopters of fundamental product, but it is actually a proportional relation, which is a function of time, we define it as the time varying proportion.

Therefore, based on PMM, we will formulate a contingent technology innovation diffusion model, 
which considering attached products diffusion with fundamental product at the same time, non-simultaneous diffusion and diffusion with dynamic time. And then simulate it by software.

\section{Theory and model}

\section{Overview of Bass model}

When they predict groceries and other easily goods, a model, which cumulative number of consumers is only related to outdoor media, television advertising and other external-influence, is proposed by Fourt \& Woodlock as follows:

$$
\frac{\mathrm{d} N(\mathrm{t})}{\mathrm{dt}}=p(M-N(t))
$$

Mansfield then presents a model that the current number of adopters is relative with internal influence between adopters and non-adopters exchange:

$$
\frac{\mathrm{d} N(\mathrm{t})}{\mathrm{dt}}=q \frac{N(t)}{M}(M-N(t))
$$

Where $N(t)$ is the number of cumulative consumers by time $\mathrm{t}, N(\mathrm{t})=\int_{0}^{\mathrm{t}} \mathrm{n}(t) \mathrm{dt}, n(t)$ is the number of new adopter at time $t$. Parameters $p$ and $q$ are innovation coefficient and imitation coefficient, respectively. $M$ denotes the maximum adopters of potential market, which is the number of adopters close to saturation of the market.

Bass combines with equations (1) and (2) and assumes potential adopters of an innovation are influenced by two types of communication channels: Mass media and interpersonal channels. Bass divides potential adopters into two groups: innovators, who are affected only by the mass media, and imitators, who are only affected by word of mouth. So basic expression of Bass model is

$$
\frac{\mathrm{d} N(\mathrm{t})}{\mathrm{dt}}=p(M-N(t))+q \frac{N(t)}{M}(M-N(t))
$$

The Bass model is based on certain assumptions including: (1) the number of potential adopters of innovation products is constant, does not change with time; (2) the diffusion of innovation product is independent of other products, and there are no other competitive products, alternative products or complementary products; (3) itself performance of the innovation product does not change with time; (4) in the whole process of diffusion, geographical boundary of innovation in social system does not change with the diffusion; (5) in the process of diffusion, there are only two kinds of selection: adopting or not adopting; (6) the diffusion process is not affected by the marketing strategy, the coefficients of innovation and imitation are constant; (7) all adopters are of homogeneous; (8) the supply of innovative products without restriction, demand can be satisfied for all; and(9) each adopter only buy a piece of the commodity, and there is no repeat purchase behavior.

\section{The derivation of contingent diffusion model}

According to above assumptions of Bass model, relaxing assumptions (1) and (2), considering the number of potential adopters of a innovation product as a variable and a product innovation is attached with it, we establish a contingent diffusion model based on dynamic time.

Perterson \& Mahajan propose a contingent diffusion model based on the Bass model according to the characteristics of attached products: 


$$
\begin{aligned}
& \frac{\mathrm{d} N_{1}(\mathrm{t})}{\mathrm{dt}}=p_{1}\left(M_{1}-N_{1}(t)\right)+q_{1} \frac{N_{1}(t)}{M_{1}}\left(M_{1}-N_{1}(t)\right) \\
& \frac{\mathrm{d} N_{2}(\mathrm{t})}{\mathrm{dt}}=p_{2}\left(N_{1}(\mathrm{t})-N_{2}(t)\right)+q_{2} \frac{N_{2}(t)}{N_{1}(\mathrm{t})}\left(N_{1}(\mathrm{t})-N_{2}(t)\right)
\end{aligned}
$$

Where equation (4) is the Bass model, as a diffusion model of fundamental product; equation (5) is the diffusion model of attached product, which is derived from the Bass model. The superior limit of potential adopters of attached product is the cumulative number of fundamental product adopters, where we note it as $N_{1}(\mathrm{t})$, which it is not a constant but it is a function of time.

But this model is only applicable to that the fundamental product and its attached products diffuse synchronously. However, after a certain period of time of the fundamental product diffusion, namely the fundamental product have a certain user group, its attached products begin to spread. Therefore, the attached product diffusion mode should take into account diffusion delay time.

Yang Jinghui and $\mathrm{Wu}$ Chunyou [12] assume attached product diffusion is late than the fundamental product diffusion for a period of time $\sigma$, and propose a contingent diffusion model as follows:

$$
\begin{aligned}
& \frac{\mathrm{d} N_{1}(t)}{\mathrm{dt}}=p_{1}\left(M_{1}-N_{1}(t)\right)+q_{1} \frac{N_{1}(t)}{M_{1}}\left(M_{1}-N_{1}(t)\right) \\
& \frac{\mathrm{d} N_{2}(\mathrm{t})}{\mathrm{dt}}=p_{2}\left(N_{1}(t+\sigma)-N_{2}(t)\right)+q_{2} \frac{N_{2}(t)}{N_{1}(t+\sigma)}\left(N_{1}(t+\sigma)-N_{2}(t)\right)
\end{aligned}
$$

Further considering the reality, the superior limit of potential adopters of attached product, i.e. $M_{2}$, is not equal to the cumulative number of fundamental product adopters at the beginning of diffusion. At the early stage of attached products derived from its fundamental product, the adopters of fundamental product have little perception of it, maybe only a few users know and want to adopt it. At this time, it is a small proportion of $M_{2}$ to $N_{1}(t+\sigma)$. With the development of information technology and the promotion of products, more and more people are concerned about the attached products. The proportion, which $M_{2}$ to $N_{1}(t+\sigma)$, rises rapidly. When attached products develop to a saturation stage, the proportion continues to increase and increasing speed is slow and reaches to one at last, i.e. $M_{2}=N_{1}(t+\sigma)$.

Through the above analysis, we can find that the proportion varies with time; its value is in the range $[0,1]$. Therefore, according to the above characteristics, we define the proportion structure as follows:

$$
\delta=\frac{M_{2}}{N_{1}(t+\sigma)}=\frac{t}{t+a}
$$

Analysis found that the equation can be, as the constraint conditions is $a>0$, and this percentage $\delta$ is increased with the increase of $t$, and finally tends to 1 , when all fundamental product adopters are attached product potential adopters. The contingent diffusion model based on dynamic time is

$$
\begin{aligned}
& \frac{\mathrm{d} N_{1}(t)}{\mathrm{dt}}=p_{1}\left(M_{1}-N_{1}(t)\right)+q_{1} \frac{N_{1}(t)}{M_{1}}\left(M_{1}-N_{1}(t)\right) \\
& \frac{\mathrm{d} N_{2}(t)}{\mathrm{dt}}=p_{2}\left(M_{2}-N_{2}(t)\right)+q_{2} \frac{N_{2}(t)}{M_{2}}\left(M_{2}-N_{2}(t)\right)
\end{aligned}
$$




$$
M_{2}=N_{1}(t+\sigma)\left(\frac{t}{t+a}\right)
$$

\section{Simulation and analysis}

\section{Both fundamental product and its attached products diffuse at the same time}

Let $\sigma=0$ and $\delta=1$, which means both fundamental product and its attached products diffuse at the same time, and by the time ${ }^{t}$, the maximum potential adopters of attached product is equal to the cumulative adopters of fundamental product. We call it basic attached product diffusion. And then we let $\sigma=0$ and $\delta=1$, which means both fundamental product and its attached products diffuse at the same time, and by the time $t$, the maximum potential adopters of attached product are $\delta$ times of the cumulative adopters of fundamental product. We call it attached product diffusion with ration coefficient. Diffusion results of fundamental product diffusion, basic attached product diffusion and attached product diffusion with ration coefficient are given in Fig.1.

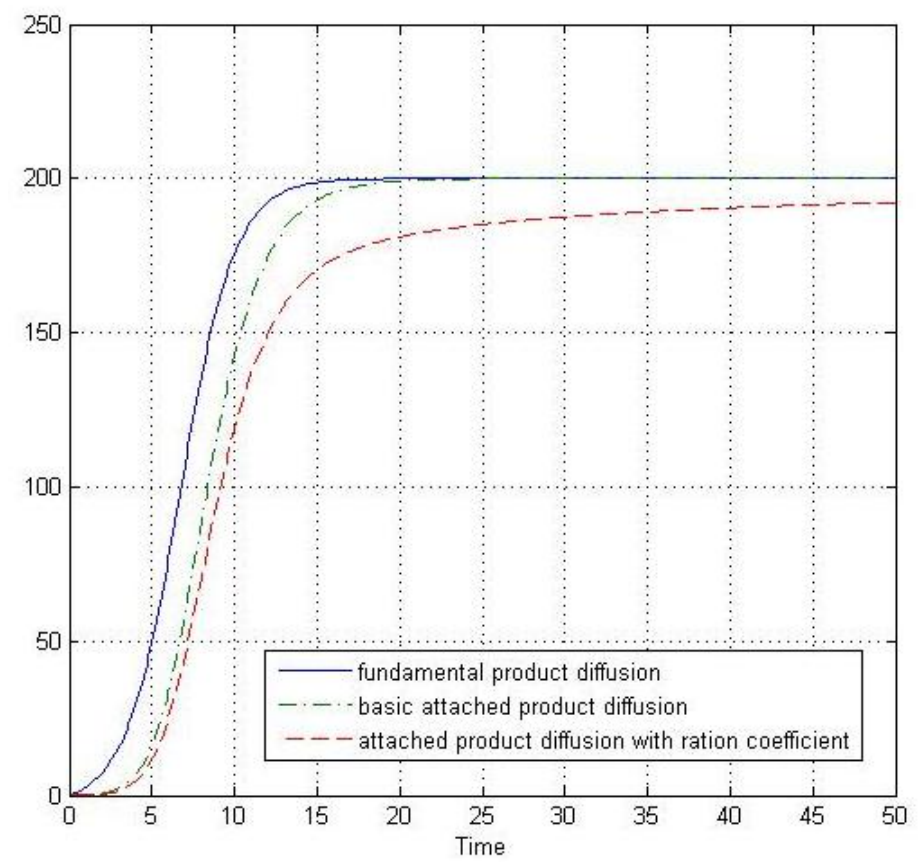

Fig.1 the cumulative adopters of three attached products

As shown in Fig.1, the adopters' curves of the fundamental product and two attached products are of shape $S$, with the passage of time they eventually reach a steady state. Before the attached product diffusion reaches the maximum scale of potential market, because of accompanying with the fundamental product diffusion, the number of attached product adopters is always less than that of its fundamental product. After the fundamental product diffusion has reached the maximum scale of potential market for a period of time, the number of attached product will reach the maximum number of potential adopters of fundamental product.

At the beginning of diffusion of attached product with a proportion coefficient, the users of fundamental product have weak perception for it, $\delta$ is small, adoption volume increases slowly. With the passage of time, the attached products have attracted more and more attention and cumulative adopters increase rapidly. When it attached to the development of product of a saturation stage, the cumulative users go into the slow growth period; the final ratio eventually reached one, at this time, which all adopters of fundamental product are potential adopters of attached product.

\section{Both fundamental product and attached products do not diffuse at the same time}

Let $\sigma=5$ and $\delta=1$, which means the attached product begin to diffuse after its fundamental 
product has diffused for five units of time, and by the time $t$, the maximum potential adopters of attached product is equal to the cumulative adopters of fundamental product. We call it attached product diffusion with delay. And then we let $\sigma=0$ and $\delta=\frac{t}{t+a}$, which means the attached product begin to diffuse after its fundamental product has diffused for five units of time,and by the time $t$, the maximum potential adopters of attached product is $\delta$ times of the cumulative adopters of fundamental product. We call it attached product diffusion with both delay and ration coefficient.

To compare attached product diffusion with delay and attached product diffusion with both delay and ration coefficient, we demonstrate their diffusion process at the same time axis (Fig.2).

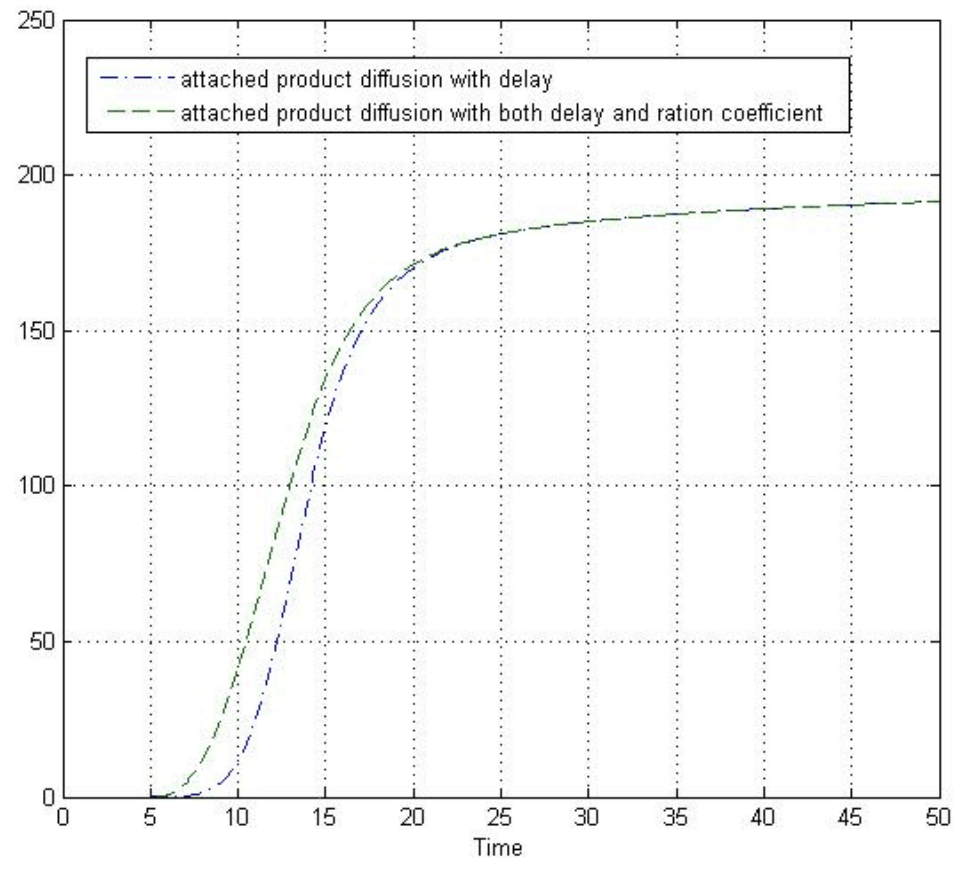

Fig.2 Contrast of cumulative adopters of two kinds of attached products

As shown from Fig.2, we find some conclusions. First, the attached product, which is late to diffuse than its fundamental product, will spread quickly at the beginning of diffusion and it is easy to accept by consumers. For example, when the number of mobile phone users is very large, Internet or mobile phone value-added service can more quickly to diffusion in the mobile phone users. Secondly, cumulative adopters of attached product diffusion with both delay and ration coefficient is greater than that of attached product diffusion with delay at the beginning and the statue lasts for a period of time and they are equal at last. So innovation companies should introduce a new product in time, and is very important for grasp a good time point to diffuse.

\section{Summary}

Considering the dependence between innovation product and its attached products, the paper tries to relax the assumptions of Bass model and establishes a contingent technology innovation diffusion model based on dynamic time, and then simulates it. The results show as follows.

(1) For the attached product diffusion, it is more realistic by using contingent diffusion model with the market potential being dynamic. However, the Bass model is more suitable for the maximum market potential being static or approximate static and it will not change within a period of time.

(2) Through the analysis of model structure and model simulation, it shows that the applicability of the contingent technology innovation diffusion model based on dynamic time, and it has a certain guiding significance to predict attached products.

(3) The attached product, which is late to diffuse than its fundamental product, will spread quickly at the beginning of diffusion and it is easy to accept by consumers. 
Though this paper has made some improvement on Bass model, there still are many shortcomings. For example, our study is only built on two layers attached diffusion relationship. In future research, not only the applicability of model need to be tested by more examples, but also more research and testing needs with multi-layer attached product model; the factors like price and advertising should be take into account in the model. In addition, follow-up study is required to determine the release time of attached products in order to make all products diffuse faster.

\section{Acknowledgement}

This research was financially supported by Social Science Foundation of Hunan Province (13YBA355).

\section{References}

[1] Schumpeter J.A. The Theory of Economic Development [M].Beijing: The Commercial Press, 2000.

[2] Rogers E M. Diffusion of Innovations [M].New York: The Free Press, 1983.

[3] Bass F M. A New Product Growth Model for Consumer Durables [J]. Management Science, 15(5) (1969):215-227.

[4] Fourt L A, Woodlock J W. Early Prediction of Early Success of New Grocery Products [J].Journal of Marketing, 25(2) (1960):31-48.

[5] Mansfield E. Technical Change and the Rate of Imitation [J].Econometrics, 29(4) (1961):741-766.

[6] Norton J A, Bass F M. A Diffusion Theory Model of Adoption and Substitution for Successive Generations of High-technology Product [J]. Management Science, 33(9) (1987):1069-1086.

[7] Mahajan V,Peterson R A. Innovation Diffusion in a Dynamic Potential Adopter Population[J]. Management Science, 24 (15) (1978):1589-1597.

[8] Bucklin L P, Sengupta S. The Co-diffusion of Complementary Innovation: Supermarket Scanners and UPC Symbols [J]. Production Innovation Management, 10(2) (1993):148-160.

[9] Yang Guozhong, Liu Congmin. Research on Dualistic Technology Innovation Diffusion Based on System Danamics [J].Soft Science, 26(8) (2012):5-10.

[10] Thakurta R, Roy R. An Investigation into Factors Influencing Penetration of Information Technology in Organizations [J].International Journal of Business Information Systems, 10(1) (2012):20-39.

[11] Sheng Ya. Technology Innovation Diffusion and New Product Marketing [M].Beijing: China Development Press, 2002.

[12] Yang Jinghui,Wu Chunyou. A Contingent Diffusion Theory Model and Its Application with Genetic Algorisms [J]. Application of Statistics and Management, 27 (2)(2007):312-317. 